\section{IRS audit for tumors}

\section{By Lev Osherovich, Senior Writer}

An Israeli academic-industry partnership has discovered small molecules that interfere with signaling by insulin-like growth factor-1 receptor, a cancer-associated receptor tyrosine kinase. The new compounds, licensed to NovoTyr Therapeutics Ltd., could be superior to conventional approaches to hitting this target because they cause destruction of two key downstream effectors, insulin receptor substrate 1 and insulin receptor substrate $2 .{ }^{1}$

Excessive activity of insulin-like growth factor-1 receptor (IGF1R; $\mathrm{CD} 221$ ) is a common feature in many tumor types. When activated by extracellular ligands such as insulin and insulin-like growth factor-1 (IGF-1), IGF1R recruits the intracellular proteins insulin receptor substrate 1 (IRS1) and IRS2 to relay a proliferative signal to a range of downstream pathways that drive tumor growth. ${ }^{2}$

Most compounds that target IGF1R are mAbs and have had a poor track record in the clinic. In 2010, Pfizer Inc.'s figitumumab (CP-751,871; CP-751871) failed a Phase III trial in advanced nonadenocarcinoma non-small cell lung cancer (NSCLC). In 2012, Amgen Inc. and Takeda Pharmaceutical Co. Ltd. terminated a Phase III trial of ganitumab (AMG 479) in pancreatic cancer owing to lack of efficacy.

Figitumumab was discontinued. Ganitumab remains in Phase II testing for a range of solid tumors, as does cixutumumab (IMC-A12) from Eli Lilly and Co.

The most advanced small molecule IGF1R inhibitor is Astellas Pharma Inc.'s linsitinib (ASP7487), which is in Phase III trials for adrenocortical carcinoma.

NovoTyr, a spinout from the laboratory of Alexander Levitzki, a professor of biochemistry at The Hebrew University of Jerusalem, initially set out to find selective kinase inhibitors of IGF1R but instead came across compounds that led to the hyperphosphorylation and degradation of IRS1 and IRS2.

"We were looking for inhibitors that compete for the substrate binding domain of IGF1R," said Levitzki. "We found that some of these molecules are in fact allosteric inhibitors that induce the dissociation of IRS1 and IRS2 from the receptor. This causes them to shift into the cytoplasm and be phosphorylated and then become degraded. This is a first-of-a-kind mechanism."

In principle, degradation of IRS1 and IRS2 should lead to longerlasting and more potent IGF1R pathway inhibition than conventional kinase inhibitors or mAbs. The challenge now is to show that the compounds perform better than other therapeutic candidates and are well tolerated.

\section{Taxing tumors}

Levitzki's team used SAR studies to identify compounds that blocked the enzymatic activity of full-length IGF1R. Surprisingly, the team found that the compounds did not block binding of ATP or inhibit the activity of a shortened version of IGF1R that contains only its kinase domain.

On the basis of this enzymological profile, the team suspected that the compounds were allosteric inhibitors that bind far from a target's active site but cause structural changes that alter the target's activity.

In a variety of cultured tumor cells, the best of the compounds inhibited tumor growth and led to hyperphosphorylation of IRS1 and IRS2 at serine residues whose phosphorylation is known to inhibit the proteins' activity. Over time, cells containing hyperphosphorylated IRS1 and IRS2 proteins underwent degradation, whereas vehicle-treated controls did not.

The group used a range of pharmacological tools to uncover the compounds' mechanism of phosphorylating and degrading IRS1 and IRS2 and found that it was mediated by the MAP kinase 1 (MAPK1; ERK-2) and MAPK3 (ERK-1) pathway.

In mouse xenograft models for melanoma, ovarian cancer and prostate cancer, the best of the compounds inhibited tumor growth and increased survival compared with no treatment.

The team did not report a head-to-head comparison of its compounds with other IGF1R inhibitors. Results were published in Cancer Research. The compounds are covered by pending patents that have been licensed to NovoTyr.

\section{Mixed signals}

Levitzki and Hadas Reuveni, study coauthor and NovoTyr's CEO, said that the principal advantage of the new compounds over conventional IGF1R inhibitors is higher potency in vivo.

"Compared to conventional IGF1R kinase inhibitors, which are given at a high frequency of one to two times a day, only a short exposure to the NovoTyr compounds is required to gain a long-lasting effect on IRS1 and IRS2," said Reuveni.

"You don't just block the signaling; you actually eliminate the downstream signaling factor," said Levitzki. "You could have more time between doses."

Another selling point is the potential of the compounds to overcome resistance to other IGF1R-targeting compounds, including IGF1Rtargeting mAbs.

Reuveni said that tumors treated with anti-IGF1R mAbs can develop resistance by upregulating a range of related receptors, including insulin receptor (INSR) and Src. The catch is that all of those receptors rely on IRS1 or IRS2 to send a downstream signal. Thus, destroying those proteins with the NovoTyr compounds could reduce the emergence of IGF1R inhibitor resistance.

"We think of IRS1 as an adaptor protein. IRS1 participates in a lot of signaling pathways, so it could interact with other kinases," said Douglas Yee, a professor of medicine and pharmacology at the University of Minnesota Medical School and director of the University of Minnesota Masonic Cancer Center. 
"Conventional approaches to targeting IGF1R downregulate the receptor activity but don't have much of an effect on IRS1," added Yee. "To really shut off IGF-1 signaling, you really have to shut off both the IGF1R and INSR. Hitting the downstream adaptor proteins may be the way to do this."

Reuveni noted that several other cancer-associated signaling pathways use IRS1 and IRS2 to relay tumor growth signals, so the compounds could be useful beyond IGF1R-overexpressing tumors.

Along these lines, the team found the compounds prevented growth of cultured tumors resistant to Zelboraf vemurafenib (PLX4032), a small molecule inhibitor of mutant BRAF that is marketed by Roche and Daiichi Sankyo Co. Ltd. to treat metastatic melanoma. The team showed that those tumors had high expression of IRS1 and IRS2 compared with Zelboraf-sensitive cells.

\section{Cancer and metabolism}

The next step for NovoTyr is to continue preclinical toxicology studies. One potential concern is whether the molecules will show an unwanted effect on normal insulin and IGF-1 signaling in metabolism.

"Theoretically, you might expect that comprehensive blockade of the entire receptor family would have broad metabolic effects," said Michael Pollak, a professor of oncology and medicine at McGill University. "The authors' to-do list should be to rule out toxicity and, if there isn't, they should understand why, since you would expect that this would cause diabetes-like effects."

"Metabolic effects are always a concern. If this affects the function of adaptor proteins, there could be a profound effect on glucose homeostasis," said Yee. Another

\section{"It's not just another} incremental advance. This belongs on the desk of a venture capitalist. It really is novel, and it just might work."

\section{-Michael Pollak, McGill University} concern is whether there would be knock-on effects on other signaling pathways that use IRS1 and IRS2. Pollak said that tinkering with other proliferative pathways such as mammalian target of rapamycin (mTOR; FRAP; RAFT1) and protein kinase B (PKB; PKBA; AKT; AKT1) signaling can affect IRS1 and IRS2 activity, so NovoTyr's compounds might affect those pathways differently than do direct IGF1R inhibitors.

"Eliminating the IRS proteins is a very interesting but hardto-predict intervention because of all of the feedback loops that converge" on IRS1 and IRS2, said Pollak.

Despite these concerns, Pollak said that the Israeli team's approach is fundamentally different from what has been tried before. "It's not just another incremental advance," he said. "This belongs on the desk of a venture capitalist. It really is novel, and it just might work."

The team already has shown the compounds are well tolerated by mice in the short term and now plan to do long-term toxicology studies.

NovoTyr was founded in 2005, and Reuveni said that the company "has raised an overall amount of $\$ 3.5$ million from several parties, mainly the Office of the Chief Scientist in the Ministry of Economy in Israel and from Teva Pharmaceutical Industries Ltd."

She said that the Israeli government investment was provided through the Meytav Technological Incubator, which holds more than $60 \%$ of NovoTyr's equity. The other shareholders are Teva, Hebrew University, cofounders Levitzki and Reuveni and some of NovoTyr's directors.

"At the moment the company is eager to raise venture capital investment," added Levitzki.

Reuveni said that Teva declined to exercise an exclusive option to acquire NovoTyr's compounds and that the molecules are available for licensing or partnering. Reuveni declined to say why Teva had passed on the compounds.

Osherovich, L. SciBX 6(20); doi:10.1038/scibx.2013.478

Published online May 23, 2013

\section{REFERENCES}

1. Reuveni, H. et al. Cancer Res.; published online May 7, 2013; doi:10.1158/0008-5472.CAN-12-3385

Contact: Alexander Levitzki, The Hebrew University of Jerusalem, Jerusalem, Israel

e-mail: alex.levitzki@mail.huji.ac.il

2. Pollak, M. Nat. Rev. Cancer 12, 159-169 (2012)

\section{COMPANIES AND INSTITUTIONS MENTIONED}

Amgen Inc. (NASDAQ:AMGN), Thousand Oaks, Calif.

Astellas Pharma Inc. (Tokyo:4503), Tokyo, Japan

Daiichi Sankyo Co. Ltd. (Tokyo:4568; Osaka:4568), Tokyo, Japan Eli Lilly and Co. (NYSE:LLY), Indianapolis, Ind.

The Hebrew University of Jerusalem, Jerusalem, Israel

McGill University, Montreal, Quebec, Canada

Meytav Technological Incubator, Kiryat-Shmona, Israel

NovoTyr Therapeutics Ltd., Kiryat-Shmona, Israel

Office of the Chief Scientist in the Ministry of Economy,

Tel Aviv, Israel

Pfizer Inc. (NYSE:PFE), New York, N.Y.

Roche (SIX:ROG; OTCQX:RHHBY), Basel, Switzerland

Takeda Pharmaceutical Co. Ltd. (Tokyo:4502), Osaka, Japan Teva Pharmaceutical Industries Ltd. (NYSE:TEVA), Petah Tikva, Israel

University of Minnesota Masonic Cancer Center, Minneapolis, Minn.

University of Minnesota Medical School, Minneapolis, Minn. 